\title{
Current status and recommendations \\ on methicillin-resistant Staphylococcus \\ aureus infection in Latin America
}

Methicillin-resistant Staphylococcus aureus (MRSA) is an important and growing cause of infection in hospital-associated settings and, more recently, in the community, both globally and in Latin America. $^{1-3}$ Infections with MRSA and other antibiotic-resistant Gram-positive organisms are challenging to treat and impose a high burden on healthcare resources. The Latin American Working Group on Gram-positive resistance was established in 2007, with the aims of reducing this burden and improving the overall management of infections due to Gram-positive resistant bacteria in the region. The group consists of 11 physicians from Latin America with interest and expertise in these issues.

The group recognized the importance of educating the multidisciplinary healthcare community about these issues and reviewing guidelines for the control and management of Gram-positive resistant bacteria, with a view to adapting them to meet the local situation. Subgroups were established to review specific topics including surveillance and epidemiology, clonal evolution, laboratory issues, and MRSA control and treatment, with the intention of addressing these goals. The papers in this supplement summarize the outcomes of these initiatives.

The first paper by Mejía et al. focuses on the epidemiology of MRSA; the authors highlight the importance of accurate surveillance information, including susceptibility data, as a tool to assist in the control and management of resistant infections. It is clear that MRSA is an important hospital pathogen across the region, and community-associated MRSA infections are also widely reported. The amount of surveillance data from Latin America are increasing, but it seems likely that infectious disease specialists do not yet make optimum use of it. It is recognized that there may be wide variations in incidence, and local data may not be representative of the local situation. Moreover, robust quality control is needed to ensure that data are reliable.
The closely related topic of the clonal pattern of MRSA is the subject of the second paper by Rodríguez-Noriega \& Seas. Strains from the main clonal complexes are widely distributed across Latin America, although their distribution and resistance profiles are varying all the time. It is also apparent that clones with increased virulence are being isolated more often from both healthcare- and community-associated infections.

Accurate diagnosis and susceptibility testing are prerequisites for appropriate treatment of MRSA infections, and these themes are picked up by Zurita et al. They emphasize the importance of guidelines for MRSA testing adapted to meet local needs and resources constraints, coordinated quality control measures and centralized reference facilities. These steps must be underpinned by educational activities and other initiatives to ensure the implementation of best practice.

Control of the spread of MRSA within hospitals and in specific populations in the community is an essential element of a strategy to reduce the burden of this infection. Alvarez et al. review current measures to prevent and control MRSA infection in Latin America, analyze the risk factors for spread of infection, and summarize key recommendations.

In the last paper in this supplement, Luna et al. review the treatment of MRSA in both hospital and community settings. They discuss the antibiotics that are available and examine the various guidelines that are available to guide practice. International guidelines provide a useful starting point; they need to be adapted in line with regional and local epidemiology, medical practices and resource constraints. Once guidelines have been agreed, ongoing educational efforts are essential to ensure that they are applied consistently in the clinical situation.

The goal of these publications is to stimulate infectious disease committees, clinicians and
Author

Eduardo Gotuzzo, on behalf of the Latin American Working Group on Gram

Positive Resistance.

${ }^{1}$ Instituto de

Medicina Tropical, Alexander von Humboldt,

Universidad Peruana Cayetano Heredia,

Lima, Peru.
Correspondence to: Eduardo Gotuzzo Head of Department of Infectious

Diseases and Tropical Medicine, Hospital Nacional Cayetano Heredia

Director, Instituto de Medicina Tropical, Alexander Von Humboldt Universidad Peruana Cayetano Heredia Av. Honorio Delgado 430, Urb. Ingenieria San Martin de Porres, Lima, Peru. Phone:

+51-1-3821021

Fax: +55-1-4823404 E-mail: eduardo. gotuzzo@upch.pe 
microbiologists across Latin America to increase their efforts to combat the rapid spread of MRSA. Comprehensive, coordinated and detailed plans are needed to reduce the burden of this increasingly prevalent and difficult infection, and healthcare professionals are encouraged to support and apply best practice in screening, prevention and treatment of MRSA.

\section{ACKNOWLEDGEMENTS}

\section{Financial support}

Pfizer Inc., New York, NY, USA, provided support for meetings of the Latin American Working Group on Gram Positive Resistance. Pfizer Inc. had no involvement in the collection, analysis and interpretation of data, in the writing of the manuscripts, or in the decision to submit the articles for publication.

\section{Manuscript preparation}

The support provided by Choice Pharma (Hitchin, UK), funded by Pfizer Inc., consisted of manuscript formatting and writing assistance.

\section{DISCLOSURES}

E. Gotuzzo: Advisory Board member and consultant for Pfizer; received research grants from Merck, Sharp \& Dohme; TIBOTEC consultant for Sanofi Pasteur.

A Spanish translation of this, the original English version of the Supplement, "Current status and recommendations on methicillin-resistant Staphylococcus aureus (MRSA) infection in Latin America" by Dr Eduardo Gotuzzo and members of the Latin American Working Group on Gram Positive Resistance, has been published in Revista Chilena de Infectologia, Vol. 27 (2), 2010. The original English version should be cited as reference.

\section{REFERENCES}

1. Woodford N, Livermore DM. Infections caused by Gram-positive bacteria: a review of the global challenge. J Infect. 2009; 59 Suppl 1:S4-16.

2. Guzmán-Blanco M, Mejía C, Isturiz R et al. Epidemiology of methicillin-resistant Staphylococcus aureus (MRSA) in Latin America. Int J Antimicrob Agents. 2009; 34(4):304-8.

3. Rodríguez-Noriega E, Seas C, Guzmán-Blanco M et al. Evolution of methicillin-resistant Staphylococcus aureus clones in Latin America. Int J Infect Dis. 2010; 14:e560-6. 